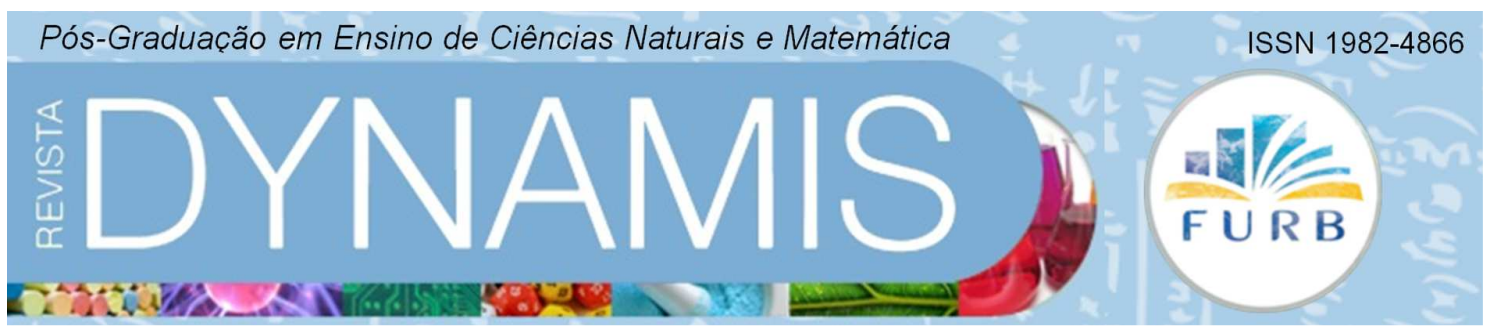

\title{
ALÉM DO QUARTO DO HOSPITAL: APRENDENDO CIÊNCIAS EM UMA CLASSE HOSPITALAR
}

Beyond the hospital room: learning science in schooling in hospital

\author{
Michele Francine Muniz Andrade \\ Universidade Regional de Blumenau \\ michelemunis@hotmail.com \\ Patrícia Leopoldo da Silva Oliveira \\ Universidade Regional de Blumenau \\ plsoliveira@hotmail.com \\ Daniela Tomio \\ Programa de Pós-graduação em Ensino de Ciências Naturais e Matemática - PPGECIM/FURB \\ Universidade Regional de Blumenau \\ dtomio@furb.br
}




\title{
Resumo
}

Em tempos em que, na construção da cidadania, é preciso incorporar no universo cultural a ciência e a tecnologia como atividades sociais, o ensino na modalidade pedagogia hospitalar representa um desafio também para o professor de ciências. Com base nesse pressuposto, relata-se uma prática de ensino de ciências desenvolvida em uma classe hospitalar. Esta é uma modalidade de educação, regulamentada pelo Ministério da Educação, que assiste estudantes na impossibilidade de frequência à escola, durante o período sob tratamento de saúde. Diante disso, apresenta-se uma experiência desenvolvida em um hospital de Blumenau/SC que consistiu no trabalho com um grupo de adolescentes internados a partir do tema "sistema esquelético e as fraturas". Foram desenvolvidas atividades relacionando as histórias de vidas dos estudantes, conhecimentos científicos e tecnológicos e uma forma de socialização dos trabalhos para além do quarto de hospital.

Palavras - chave: Ensino de Ciências. Ciência-Tecnologia-Sociedade. Classe Hospitalar.

\begin{abstract}
At a time when the construction of citizenship needs to incorporate the cultural universe of science and technology as social activities, teaching at hospital classes may also represent a challenge for a science teacher. Based on this assumption, it is reported a practice of science teaching developed in a schooling hospital. This is a form of education, regulated by the Ministry of Education, which assists students who have the impossibility to attendee school during the period in health care. Therefore, we present an experience developed in a hospital in Blumenau / SC in which consisted in a work with a group of adolescents that developed a study about the "skeletal system and fractures." It was developed a lot of activities related to the history of the adolescent's lives, scientific and technological knowledge and also, a form of work socialization beyond the hospital room.
\end{abstract}

Keywords: Science Teaching. Science-Technology-Society. Schooling in hospital. 


\section{INTRODUÇÃO}

Dentre as modalidades de educação formal está a classe hospitalar. Esta oferta de ensino é um direito, regulamentada pelo Ministério da Educação, que assiste estudantes na impossibilidade de frequência à escola, durante o período sob tratamento de saúde ou de assistência psicossocial. (BRASIL, 2002).

Diante disso o Ministério da Educação, por meio de sua Secretaria de Educação Especial, elaborou um documento de estratégias e orientações a fim de fomentar a disponibilização do atendimento pedagógico em ambientes hospitalares e domiciliares com o propósito de garantir o "[...] acesso à educação básica e à atenção às necessidades educacionais especiais, de modo a promover o desenvolvimento e contribuir para a construção do conhecimento desses educandos". (BRASIL, 2002. p.7)

Na classe hospitalar o ensino de ciências também pode ser abordado, uma vez que é um componente curricular da educação básica e os estudantes têm direito de acesso à educação científica enquanto estiverem hospitalizados. Com base nessa premissa, realizou-se uma prática educativa na Ala Pediátrica do Hospital Santo Antônio (HSA), no município de Blumenau/SC. O estudo insere-se no Projeto Pedagogia Hospitalar, que é uma iniciativa da Secretaria Municipal de Educação (SEMED) e conta com a parceria do Hospital Santo Antônio com a Universidade Regional de Blumenau (FURB) ${ }^{1}$.

No espaço do hospital, os estudantes internados realizam várias atividades, desenvolvidas para reforço, revisão ou introdução de conteúdos dos vários componentes curriculares. Estas são propostas pelas Escolas dos estudantes e no Hospital contam com uma professora e a parceria dos parente-acompanhantes para a sua realização.

Neste contexto, objetivou-se desenvolver uma prática educativa, com ênfase no ensino de Ciências, com crianças e adolescentes internados na ala pediátrica do HSA. Esta organização inseriu-se em uma proposta de estágio da licenciatura em Ciências Biológicas da FURB.

Outros estudos realizados com o objeto de investigação especificamente relacionados ao Ensino de Ciências em Classes Hospitalares podem ser destacados, como Linheira (2006, p. 15) que objetivou "investigar a existência de elementos do ambiente hospitalar que poderiam ser pontos de partida para contextualização do ensino de ciências em classes hospitalares do ponto de vista da aceitação e da curiosidade dos alunos pacientes". Nesta direção, Santos (2008) buscou aprofundar temas importantes para o campo do ensino de ciências da classe hospitalar em Florianópolis/SC, considerando as rotinas e procedimentos que os jovens vivenciam durante a internação com ênfase nos processos de aprendizagem.

Neste conjunto, esta prática educativa justifica-se em tempos em que, na construção da cidadania, é preciso incorporar no universo cultural a ciência e a tecnologia como atividades sociais, com origens e fins sociais, compromissados politicamente com a sociedade. Assim o ensino na modalidade pedagogia hospitalar representa um desafio também para o docente de Ciências.

${ }^{1}$ PEDAGOGIA HOSPITALAR - HOSPITAL SANTO ANTONIO: SEMED BLUMENAU/FURB. Disponível em: <http://pedagogiahospitalarfurbhsa.blogspot.com.br/ >. Acesso em 10 abr. 2014. 


\title{
2 APRENDER CIÊNCIAS NA EDUCAÇÃO HOSPITALAR
}

O que busca a classe hospitalar e de que ensino de ciências se está defendendo nestes espaços e tempos de aprender? Vasconcelos $(2004$, p. 1) destaca que "a classe hospitalar é o ambiente que deve buscar recuperar a socialização da criança por um processo de inclusão, dando continuidade a sua aprendizagem".

A classe hospitalar, um espaço de ensino pedagógico-educacional em hospitais, “[...] surge como um contexto que permite flexibilizar a exploração do cotidiano de seus estudantes (jovens internados) e a promoção de novas estratégias de ensino-aprendizagem de forma mais imediata”. (SANTOS, 2008, p. 2)

Ao se considerar a classe hospitalar como um espaço de aprendizagem concorda-se com Ceccim (1999, p. 4 grifo nosso) de que é:

\begin{abstract}
[...] urgente e necessária a expansão e a melhoria da educação em classes hospitalares em nosso país, aliada aos valores da cidadania, ao direito de educação e ao direito de saúde, com a clareza de que o direito de educação passa pelo reconhecimento das necessidades e do direito ao atendimento pedagógicoeducacional e que o direito de saúde passa pelo reconhecimento de direito ao atendimento das necessidades intelectuais e sócio-interativas da infância. O principal efeito do encontro educação e saúde para uma criança hospitalizada é a proteção do seu desenvolvimento e a proteção dos processos cognitivos e afetivos de construção dos aprendizados.
\end{abstract}

Com base nesses autores, parte-se do pressuposto de que o trabalho com educação científica no espaço da educação hospitalar precisa, além do rigor com os objetivos de aprendizagem, incentivar o estudante-paciente a compreender a ciência como parte de sua cultura e o seu papel, como cidadão, nas discussões relacionadas a este conhecimento.

Entende-se que os estudantes internados quando valorizados como sujeitos aprendentes que se perguntam e consomem diariamente produtos da ciência e da tecnologia podem questionar como são as suas relações com o mundo. Compartilhando dessa ideia com Tomio (2012, p. 158):

Desde cedo, o ensino de ciências pode contribuir para as crianças perceberem o significado social dos saberes científicos e tecnológicos em suas ações do cotidiano ao conhecerem, por exemplo, os modos de produção desses conhecimentos ao longo da história e na atual sociedade em que vivem. Também, motivá-las para o gosto de continuar a aprender, com autonomia e crítica, sobre ciência e tecnologia, além da escola, tornando-se jovens/adultos que visitam espaços informais de educação científica, selecionam e leem revistas, jornais, sites, programações de TV, entre outros.

Nesta direção, é de se esperar que as crianças e adolescentes que precisam estar em um hospital para tratamento de saúde, encontrem neste espaço e tempo processos educativos intencionais que contribuam para ampliarem as suas formas de pensar a fim de indagarem (se) e estabelecerem relações cada vez mais complexas entre conhecimentos e processos científicos e tecnológicos para que continuem curiosos e interessados em aprender, que 
anseiem e possam participar democraticamente de processos que envolvam decisões para suas vidas, principalmente ligadas à sua saúde. (TOMIO, 2012).

Além disso, que os sujeitos que aprendem, mesmo nas classes hospitalares, tenham condições para questionar os sentidos naturalizados para os conhecimentos científicos e tecnológicos e suas relações com o desenvolvimento de nossa sociedade:

[...] nos aspectos mais particulares daquelas atividades cujos produtos insinuam-se de maneira quase imperceptível, mas decisiva, nos mais íntimos espaços de nossas vidas, de nossos pensamentos e modos de ser que, de tão próximos, parecem naturais e inquestionáveis. (LINSINGEN, 2007, p. 17)

Com base nessas considerações sobre o aprender ciências, como organizar práticas educativas em classes hospitalares considerando as relações entre Ciência, Tecnologia e Sociedade?

\section{PROCEDIMENTOS METOLÓGICOS}

\subsection{O CONTEXTO}

O Hospital Santo Antônio (HSA), localizado no município de Blumenau / SC foi inaugurado em 1860, quando o fundador da cidade Dr. Hermann Bruno Otto Blumenau viu a necessidade de se criar um espaço para atendimento médico hospitalar com qualidade para a população da Região do Vale do Itajaí. Hoje é referência em várias especialidades médica oncologia, ortopedia e materno-infantil para a região do Vale do Itajaí. A ala pediátrica do HSA é referencia no estado em qualidade de atendimento, equipe e por contar com o Projeto Pedagogia Hospitalar.

O HSA conta com uma classe hospitalar e professoras pedagogas para o atendimento dos estudantes internados. Estes recebem atendimentos educacionais nos próprios leitos ou no espaço de aprender, sala onde todas as crianças e adolescentes podem interagir com conversas e atividades propostas pelas professoras, como o acompanhamento pedagógico.

As crianças e adolescentes recebem também atendimentos de especialistas da saúde, acompanhamento de psicólogos, pediatras, enfermeiros e estagiários de licenciaturas da FURB. Os pais das crianças podem acompanhá-los nas atividades propostas pelas professoras, dando auxílio e, muitas vezes, auxiliando-os nas atividades.

Nesta realidade foi realizada uma prática educativa em que participaram no total de 17 estudantes de 9 a 14 anos, internados entre os meses de setembro a novembro do ano de 2012 . Os estudantes internados eram de diferentes escolas, todos da rede pública de ensino e compreendidos na maioria entre os anos/séries de $6^{\circ}$ ao $9^{\circ}$ ano.

\subsection{A PRÁTICA EDUCATIVA, EM ENSINO DE CIÊNCIAS, EM UMA CLASSE HOSPITALAR}

A rotina da Ala Pediátrica do Hospital é totalmente distinta de uma unidade escolar, uma vez que exigem formas de trabalho flexíveis e diversas que possam atender a diversidade de estudantes, suas exigências educacionais e limites de estudar em função de suas doenças. 
Os estudantes recebem atendimentos educacionais nos leitos ou em um espaço de aprender, onde podem interagir com os outros. Neste cenário, organizou-se uma experiência de aprendizagem de Ciências que possibilitou incluir a todos internados no período de três meses.

Para tal, partiu-se de um tema de estudo, selecionado em parceria com a professora do Hospital e com coordenadoras responsáveis pelo projeto da SEMED. O tema foi "sistema esquelético e as fraturas", que surgiu a partir da campanha da semana do trânsito no município e a incidência de fraturas das vitimas de acidentes de trânsito. Também, considerou-se um levantamento da causa de maior internação das crianças no período em que foi realizada a prática de estágio.

A partir do tema, foram elaboradas com os estudantes atividades, como a montagem de um esqueleto do corpo humano, com a identificação dos principais ossos a partir de consulta em um de atlas do corpo humano (fig. 1); a discussão temas relacionados à função dos ossos, as fraturas e como o osso se regenera. Ainda, a produção de um gênero textual, cujo propósito foi relacionar as histórias de vidas dos estudantes, conhecimentos científicos e tecnológicos e uma forma de socialização dos conhecimentos elaborados para outras pessoas, além dos muros do hospital.

Figura 1 - Registro da atividade de montagem do esqueleto

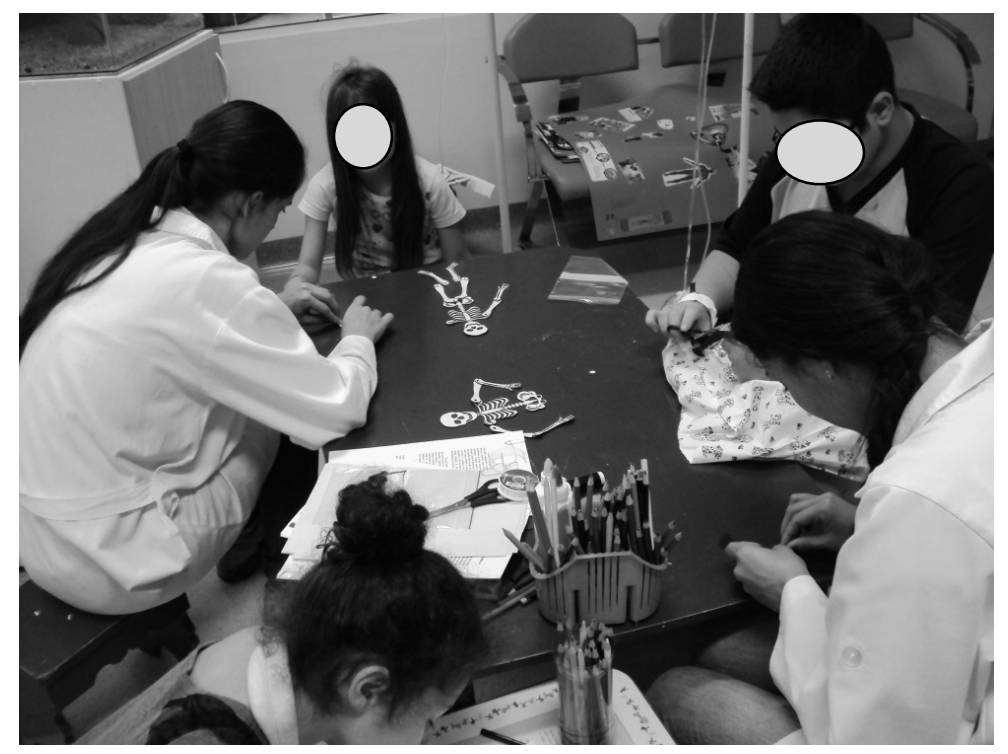

Fonte: Arquivo das autoras

Para fechamento das atividades, elaborou-se com os estudantes internados um gênero textual no formato de um "encarte", que teve como inspiração a leitura de outro texto desse gênero divulgado por uma rede de alimentos que faz uma campanha, um dia do ano, para arrecadar fundos para o tratamento de crianças com câncer.

A partir disso, coletaram-se textos, produzidos em conjunto com os estudantes e seus acompanhantes, sobre suas histórias de vidas relacionadas à doença que motivou a internação. Agregaram-se informações científicas sobre o corpo humano, principalmente sobre fraturas e formas de regeneração óssea. Também, sugeriram-se textos de entrevistas com especialistas 
do hospital sobre formas de tratamento e de prevenção dessas doenças. O texto final, o encarte, também foi ilustrado, impresso, e teve como finalidade socializar a experiência de internação para outras pessoas, além do quarto do hospital.

Além dos conteúdos conceituais próprios do estudo do corpo humano, objetivou-se incentivar os estudantes a desenvolverem conteúdos procedimentais como: elaborar informes científicos e outras formas de socialização de conhecimentos por escrito; identificar informações de folders e campanhas de informações ligadas à saúde; observar situações cotidianas e relacioná-las com os assuntos estudados.

Buscou-se trabalhar conteúdos atitudinais, como divulgar informações à família e outros grupos sociais sobre o papel de todos na manutenção da saúde; responsabilizar-se em relação à saúde individual e o cuidado com seu corpo; adotar formas de prevenção de doenças, dentre outros.

Observou-se que os estudantes demonstraram muito interesse na realização das atividades e elaboração conceitual em relação seus conceitos iniciais.

\section{CONSIDERAÇÕES FINAIS}

Em tempos em que, na construção da cidadania, é preciso incorporar no universo cultural a ciência e a tecnologia como atividades sociais, com origens e fins sociais, compromissados politicamente com a sociedade, o ensino na modalidade pedagogia hospitalar representa um desafio também para o docente de Ciências. Com este desafio, amplia-se, também, a necessidade de se explorar aspectos e as especificidades desse cenário de aprendizagem na formação de professores. Esta constatação tem, também, por base as inserções do Programa de Extensão "Educação em Ciências para o Século XXI" (desenvolvido na Universidade Regional de Blumenau), que desenvolve ações de apoio teórico e metodológico de professores em diferentes contextos de Educação Científica.

Pode-se contatar que atuar no lugar de docente em uma classe hospitalar requer, além do conhecimento da área de ciências e do conhecimento didático, saberes específicos desse contexto. Com os resultados da prática educativa realizada pode-se concluir que o planejamento das atividades em ensino de Ciências precisa ter o rigor do compromisso dos objetivos educacionais, porém precisa ter a flexibilidade para atender estudantes de diferentes anos, patologias distintas, das expectativas dos familiares, buscando sempre que os pais interajam e participem das atividades com os filhos, além da rotatividade das internações e o cumprimento das rotinas do hospital.

Ao observar cada paciente-estudante durante as atividades podem-se entender principalmente as palavras de Ceccim (1999, p. 14), quando escreve que ao dispor do atendimento em classe hospitalar, mesmo que por um tempo mínimo, permite a este sujeito "desvincular-se, mesmo que momentaneamente, das restrições que um tratamento hospitalar impõe e adquirir conceitos importantes tanto à sua vida escolar quanto pessoal, [...] e podendo sentir que continua aprendendo e indo à escola, portanto, renovando seu ser criança e renovando potências afirmativas de invenção da vida".

Nesta direção, observa-se com o estudo que um dos principais resultados da relação entre educação tecnocientífica e saúde para uma criança/adolescente hospitalizada é a possibilidade da construção de aprendizados. Pode-se concluir que no espaço e tempo em que estudantes estão internados é possível vivenciarem práticas educativas que lhes favoreçam o desenvolvimento da atitude curiosa e reflexiva para suas práticas no cotidiano e a elaboração 
de conhecimentos científicos e tecnológicos que contribuam para a explicação de fenômenos de sua realidade, como os de saúde que estão enfrentando no tempo de internação no hospital. Ainda, destaca-se a relevância dessas práticas como estágio curricular na formação inicial do professor de Ciências.

Agradecimentos: À professora Elenir Reeder Buddag - Coordenadora na FURB do Projeto de Extensão Pedagogia Hospitalar; à professora Betty P.R. Garcete e à pedagoga Fabiana Goldmann da Classe Hospitalar do Hospital Santo Antônio de Blumenau/SC pela oportunidade e parceria na realização do estágio que deu origem à investigação e à professora Vera Lúcia de Souza e Silva que compartilhou das experiências no estágio.

\section{REFERENCIAS}

BRASIL. Secretaria de Educação Fundamental. Parâmetros Curriculares Nacionais: Saúde. Brasília: MEC; SEF, 1998.

BRASIL. Secretaria de Educação Especial. Classe hospitalar e atendimento pedagógico hospitalar estratégias e orientações. Brasília: MEC; SEESP, 2002.

CECCIM, R. B. Classe hospitalar encontros da educação da saúde no ambiente hospitalar. Pátio, Porto Alegre, n.10, ago/out. 1999.

FONTES, R. S. A classe hospitalar e a inclusão da criança enferma na sala de aula regular. Revista Brasileira de Educação Especial, São Paulo, v.8, n.1, 2002.

LINHAIRA, C. Z.. O Ensino de Ciências na Classe Hospitalar: Um Estudo de caso do Hospital Infantil Joana de Gusmão, Florianopolis/SC. 2006. Dissertação (Mestrado em Educação Cientifica e Tecnológica) - Universidade Federal de Santa Catarina, Florianópolis, 2006.

LINSINGEN, I. von. Perspectiva educacional CTS: aspectos de um campo em consolidação na América Latina. Ciência \& Ensino, Campinas, v. 1, p. 1-19, 2007. Disponível em: $<$ http://www.ige.unicamp.br/ojs/index.php/cienciaeensino/article/view/ 150/108>. Acesso em: 11 nov. 2013.

MATOS, E. L. M.; MUGIATTI, M. M. T. de F.. Pedagogia hospitalar: a humanização integrando educação e saúde. 2. ed. Petrópolis: Vozes, 2007.

MAtos, E. L. M.; MugiATti, M. M. T. de F.. (Org.). Teoria e Prática na Pedagogia Hospitalar: novos cenários, novos desafios. Curitiba: Champagnat, 2010.

MOHR, A. A natureza da educação em saúde no ensino fundamental e os professores de ciências. 2002. Tese (Pós-Graduação em Educação) - Universidade Federal de Santa Catarina, Florianópolis 2002. 
PEDAGOGIA HOSPITALAR - HOSPITAL SANTO ANTONIO: SEMED BLUMENAU/FURB. Disponível em: <http://pedagogiahospitalarfurbhsa.blogspot.com.br/ >. Acesso em 10 abr. 2014.

SANTOS, D. Aprendizado adquiridos no hospital: análise para um ensino de ciências na classe hospitalar. Dissertação (Programa de Pós-graduação em Educação Científica e Tecnológica) - Universidade Federal de Santa Catarina, Florianópolis, 2008.

TOMIO, D. Circulando sentidos, pela escrita, nas aulas de Ciências: Com interlocuções entre Fritz Müller, Charles Darwin e um coletivo de estudantes. 2012. Tese (Doutorado) Curso de Pós-graduação em Educação Científica e Tecnológica, Universidade Federal de Santa Catarina, Florianópolis, 2012.

VASCONCELOS, S.M.F. Classe Hospitalar no Mundo: Um desafio à infância em sofrimento. Disponível em: <http://www.secult.salvador.ba.gov.br/SITE/documentos/ espacovirtual/espaco-educacao-saude/classes-hospitalaresEBARTIGOS/classe $\% 20$ hospitalar $\% 20$ no\% 20mundo.pdf>. Acesso em: 15 abr. 2014. 